\title{
Functional In Vitro Studies Of Buffy Coat Pooled Platelets Cryopreserved In Dimethyl-Sulphoxide With a New
}

\section{System}

1. Mariasanta Napolitano, MD*, ,

2. Lucio Lo Coco*,2,

3. Giorgia Saccullo, MD*, ,

4. Giovanni De Francisci, MD*,3,

5. Amalia Reina, $\mathrm{PhD}^{*}, 4$,

6. Debora Allegro, PhD*,4,

7. Raffaella Fadda, MD*,

8. Diana Di Liberto, $\mathrm{PhD}^{*}, 5$,

9. Salvatrice Mancuso, MD*,

10. Luca Valore, MD*, ,

11. Gianpaolo Vaccarella*, 6 ,

12. Rosalia Agliastro, MD*,3,

13. Francesco Dieli*, ${ }^{5}$, and

14. Sergio Siragusa, MD

\begin{abstract}
Introduction Cryopreservation for long term storage of platelets (PLTs) represents a clinical useful method for avoiding platelet shortage. Many studies have tried to define, in vitro and in vivo, the entity and weight of storage-related PLTs lesions with discordant results related to different methods.

We have performed an in vitro prospective study to evaluate PLTs count, viability and function of buffy coat derived pooled platelet concentrates (BC-PLTs) treated with dimethyl-sulphoxide (DMSO) and cryopreserved at $-80^{\circ} \mathrm{C}$ with an innovative patented system not requiring laminal flow hoods and external manipulations.

Materials and methods Each BC-PLTs was obtained from 5 buffy coats and pooled according to standard procedures. The final PLTs concentrates were leukoreduced by filtration and transferred to a $650 \mathrm{~mL}$ cryopreservation kit (Promedical ${ }^{\circledR}$ ) which allowed mixing with DMSO $25 \%$ in a closed system and following removal of supernatant without further manipulations. BC-PLTs were washed prior freezing with removal of at least $84 \%$ supernatant solution, suspended in homologous plasma from 1 of the 5 donors to a final concentration of $200 \mathrm{~mL}$ and frozen at $-80^{\circ}$. Selection criteria to make BC-PLTs available for this study was pooled PLTs concentration $>1250 \times 10^{9} / \mathrm{L}$ and a blood units collection time duration shorter than 6
\end{abstract}


minutes. All the 245 donors were healthy volunteers and they did not take any medication affecting PLTs function. BC-PLTs were analyzed immediately pre-freezing (T0) and 3 months after cryopreservation ( CRY BC-PLTS).

The following parameters were assayed: PLTs count (PC), mean platelet volume (MPV), $\mathrm{pH}$, flow cytometry (FACS) expression of CD41a, CD42b, CD61a, CD62p, PAC-1, Annexin V PLTs surface antigens and thromboelastography (TEG). All samples were analyzed also after dilution (1:4) with homologous plasma to approximately $400 \times 10^{9} /$ L PLTs (data not shown) and for bacterial contamination (BC). CRY BC-PLTs samples were thawed in a bath at $37^{\circ} \mathrm{C}$ for 5 minutes and evaluated promptly. All the tests were performed according to current European recommendations. PLTs swirl was furthermore visually assessed. Results were expressed as mean +/- standard deviation (SD). Results obtained at T0 and after 3 months were compared by paired sample t-test. Differences were considered as significant at $p$ values $<0.05$.

Results In vitro cell parameters were measured on 49 BC-PLTs and 15 CRY BC-PLTs as reported in Table1. PC was only slightly reduced in CRY BC-PLTs while MPV was significantly increased in CRY BC-PLTs. There were no differences between the 2 groups in CD41a, CD61a, CD62p and Annexin V expression while a significant reduction in CD 42b, PAC-1 for CRY BCPLTs was observed. TEG parameters were all significantly reduced in CRY BC-PLTs samples without affecting hemostasis. PLTs swirl was observed in all samples and BC was absent. View this table:

- View popup

- View inline

Table 1

In vitro assays of pre-freeze and cryopreserved platelets

Conclusions The preliminary results of our investigation confirm the potential of a new system to overcome limits to PLTs storage. In fact, this method guarantees sterility and avoids excessive manipulations thanks to a closed system. Our method avoids PLTs apoptosis, as shown by absent expression of Annexin V. The adequate hemostasis achieved at TEG in both groups supports the hypothesis that in vitro PLTs activation/deterioration doesn't necessarily mirror an impaired hemostatic in vivo function of CRY BC-PLTs. The next step of current study will be to evaluate the influence of the observed in vitro activation changes on PLTs recovery and survival in vivo.

Disclosures: De Francisci: Promedical: Consultancy. Reina: PROMEDICAL: Consultancy. Allegro: Promedical: Employment. 